\title{
Pseudo-colour with K-means Clustering Algorithm for Acute Ischemic Stroke Lesion Segmentation in Brain MRI
}

\author{
Abang Mohd Arif Anaqi Abang Isa ${ }^{1 *}$, Kuryati Kipli ${ }^{1}$, Ahmad Tirmizi Jobli ${ }^{2}$, \\ Muhammad Hamdi Mahmood ${ }^{2}$, Siti Kudnie Sahari ${ }^{1}$, Aditya Tri Hernowo ${ }^{3}$ and \\ Sinin Hamdan ${ }^{1}$ \\ ${ }^{1}$ Faculty of Engineering, Universiti Malaysia Sarawak, 94300 Kota Samarahan, Sarawak, Malaysia \\ ${ }^{2}$ Faculty of Medicine and Health Science, Universiti Malaysia Sarawak, 94300 Kota Samarahan, Sarawak, Malaysia \\ ${ }^{3}$ Faculty of Medicine, Universiti Malaya, 50603 Kuala Lumpur, Malaysia
}

\section{ABSTRACT}

Segmentation of an acute ischemic stroke from a single modality of a greyscale magnetic resonance imaging (MRI) is an essential and challenging task. Recently, there are several numbers of related works on the automatic segmentation of infarct lesion from the input image and give a high accuracy in extraction of infarct lesion. Still, limited works have been reported in isolating the penumbra tissues and infarct core separately. The segmentation of the penumbra tissues is necessary because that region has the potential to recover. This paper presented an automated segmentation algorithm on diffusion-weighted magnetic resonance imaging (DW-MRI) image utilizing pseudo-colour conversion and K-means clustering techniques. A greyscale image contains only intensity information and often misdiagnosed due to overlap intensity of an image. Colourization is the method of adding colours to greyscale images which allocate luminance or intensity for red, green, and blue channels. The greyscale image is converted to pseudo-colour is to intensify the visual perception and deliver more information. Then, the algorithm segments the region of interest (ROI) using K-means clustering. The result shows

ARTICLE INFO

Article history:

Received: 14 September 2020

Accepted: 30 December 2020

Published: 30 April 2021

DOI: https://doi.org/10.47836/pjst.29.2.03

$\overline{\text { E-mail addresses: }}$

abganaqi2@gmail.com (Abang Mohd Arif Anaqi Abang Isa)

kkuryati@unimas.my (Kuryati Kipli)

jatirmizi@unimas.my (Ahmad Tirmizi Jobli)

mmhamdi@unimas.my (Muhammad Hamdi Mahmood)

sskudnie@unimas.my (Siti Kudnie Sahari)

hernowo@um.edu.my (Aditya Tri Hernowo)

hsinin@unimas.my (Sinin Hamdan)

* Corresponding author the potential of automated segmentation to differentiate between the healthy and lesion tissues with $90.08 \%$ in accuracy and 0.89 in dice coefficient. The development of an automated segmentation algorithm was successfully achieved by entirely depending on the computer with minimal interaction.

Keywords: Acute ischemic stroke, clustering, MRI, pseudo-colour, segmentation 


\section{INTRODUCTION}

Stroke is part of the cardiovascular diseases and can be subtyped into ischemic stroke and hemorrhage stroke. Magnetic Resonance Imaging (MRI) technology is utilized extensively in the identification of brain pathologies (Gonzalez \& Schawmm 2016). When the patient positioned in a strong magnetic field, magnetization occurs in the tissue. It produces Radio Frequency (RF) signals that are released by the tissue hence creating the MR image. MRI is better than x-ray, Computed Tomography (CT), and ultrasound in differentiating a diseased and healthy areas of the brain.

Currently, radiologists are required to manually delineate the image slices consecutively to detect the diseases of the human brain in MR images, and this considered as timeconsuming. Each slice of the medical images is a valuable data source and may determine the outcome of the treatment for the patient. Incorrect segmentation would lead to inaccurate diagnosis and delaying treatment of the disease. Improper segmentation could result in a poor outcome for the patient. Thus, the automated processing of the vast image data source is necessary to overcome these issues.

Challenges exist in developing automated acute ischemic stroke lesion segmentation as the detection of the stroke area on MRI is influenced by the different signal intensities of MRI. Signal strength changes over a period in acute infarction and differs across various magnetic strength of the machine (Merino \& Warach 2010, Purushotham et al., 2015). Another issue in developing an automated segmentation from greyscale images from brain MRI is the similarities of the images of diseased areas to the healthy brain. The greyscale images of infected areas range in the grey intensity, thus making it difficult to distinguish the healthy brain tissues with the diseased areas.

Pseudo-colour image processing is a technique that allocates colours to grey values of a grayscale image based on specific indicators. This procedure is computationally simple and fast. The pseudo-colour translation is a useful enhancement technique to transform a greyscale image, therefore, improving the capability of distinguishing the image information which has broadly applied in medical, engineering, and military fields (Jinlong et al., 2012). Meanwhile, K-means clustering segmentation classified under pixelbased segmentation methods, whereby the application of this method is more practical for biomedical images. The number of clusters in the biomedical image generally recognized for images of sections of the human anatomy.

Recently, there are numerous researches on brain MRI segmentation of the acute ischemic stroke. Muda et al. (2017) discussed an automated segmentation approach of brain lesions from DWI by employing the Fuzzy C-means (FCM) segmentation algorithm. The clustering algorithm achieved by merging the spatial neighborhood data points with the standard FCM algorithm. Nag et al. (2017) proposed a segmentation technique based on the Gaussian mixture model (GMM) for the localization of stroke lesions in DWI 
images. The segmentation accomplished when several components of GMM generate a multimodal density, and clusters were assigned to maximize the maximum likelihood function. Vupputuri et al. (2017) presented a hierarchical region splitting (HRS) approach to delineate acute ischemic stroke lesions area using diffusion- and perfusion-weighted image (DWI-PWI) maps from adult patients. They rejected outliers of ROIs from several image maps by cross-validation and sensibly fused to generate final ROIs by utilizing the statistical technique.

Meanwhile, Gautam and Raman (2019) introduced the histogram-based thresholding (HT) and random forest-based (RF) brain classification to segment the stroke lesion. A clustering segmentation on greyscale MRI image has been done by Dhankar et al. (2010) and Nitta et al. (2020). In work by Dhankar et al. (2010), the quality of the segmentation was increased with the increasing value of the number of centroids, $k$ and many $k$ were formed, and it was difficult to recognize the segmented regions. Besides, there were 16 probabilities calculated for initial centroids in work by Nitta et al. (2020). These high number of centroids calculated hence increase the computational cost in the k-means clustering segmentation approach.

In this research, we will propose an image segmentation algorithm that consists of several parts. The core part is the conversion of grayscale MRI into the pseudo-colour combined with a clustering algorithm. The proposed approach will help medical experts to differentiate acute ischemic stroke lesion in structural MRI brain image. The MRI modality used in this research is the Diffusion Weighted Image (DWI), and we focus on hyperintense lesions. The critical contribution of this method is that the stroke lesion can be distinguished from healthy brain tissue by enhancing a greyscale image to colourize image. In summary, the contributions of this paper include 1) development of the automatic segmentation algorithm for acute ischemic stroke lesion in structural MRI brain image. 2) validation and evaluation of the performance of the segmentation algorithm against the ground truth.

This paper is organized as follows. In section II will describes the methods and algorithms of this segmentation. Meanwhile, section III presents the experimental results and discusses them. Lastly, the conclusions are given in Section IV.

\section{METHODS}

In this section, we present a pseudo-colour conversion with k-means clustering algorithm method for acute ischemic stroke lesion segmentation on the diffusion-weighted image. The flowchart of the proposed methodology of the segmentation algorithm is displayed in Figure 1. We divided the segmentation flow into three processes, namely pre-processing, pseudo-colour translation, and segmentation. Each approach gives a significant contribution to the segmentation outcome. 


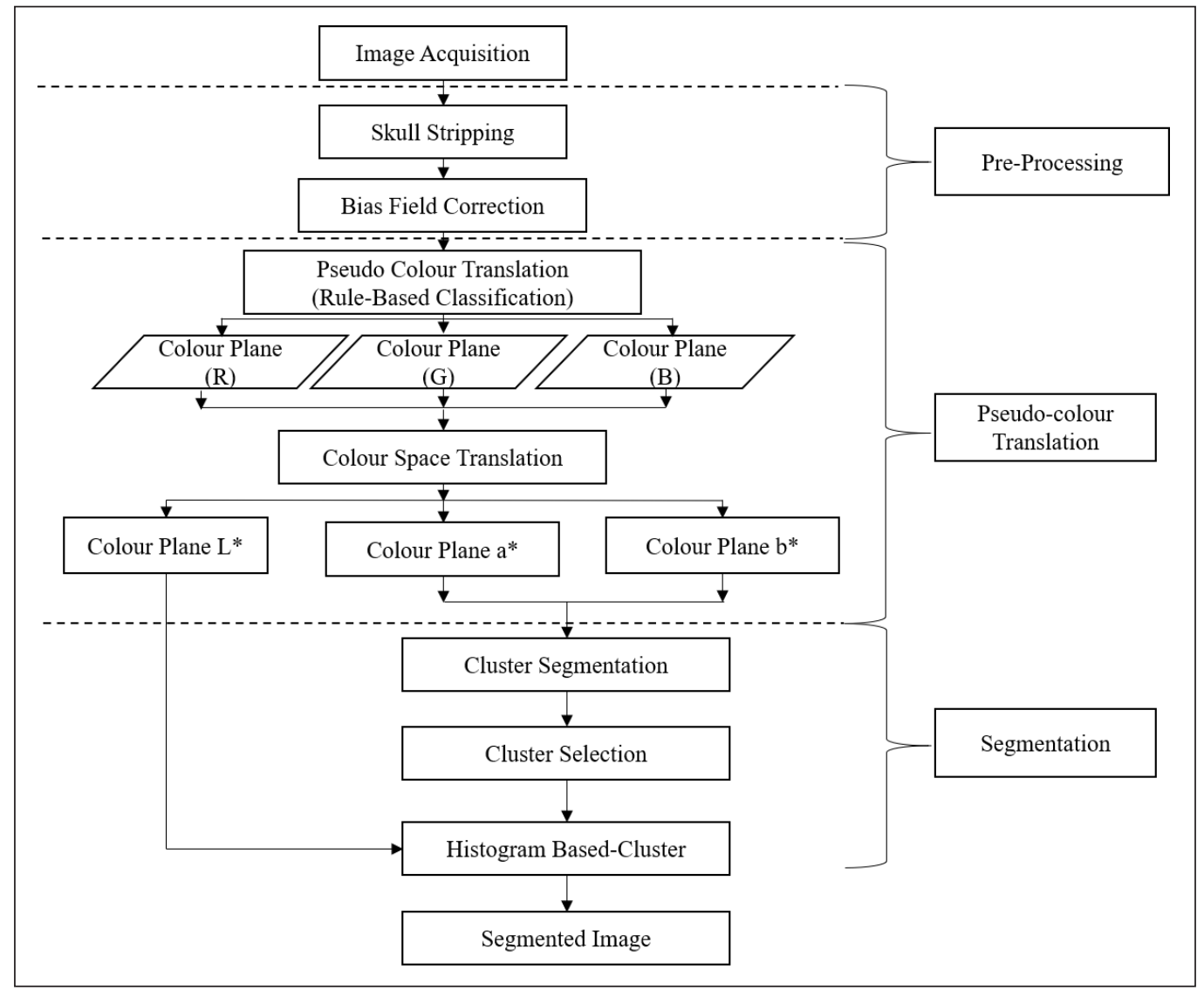

Figure 1. Process flow of the proposed method

Image pre-processing is one of the primary steps in most of the segmentation of the medical image. This step is highly essential to ensure the high accuracy of the segmentation. MR brain images generally consist of some visual artefacts. These artefacts in MRI may be caused by the equipment or the patient itself (Graves \& Mitchell, 2013). Interference of the field homogeneity, because of the magnetic material inside or outside of the scanner equipment often occurred while taking images from the patients, and patient-specific artefacts include brain skull and motion artefacts caused by breathing and movement (Krupa \& Bekiensinska-Figatowska, 2015). These artefacts need to be eliminated by the pre-processing process before continuing to the subsequent steps.

A pseudo-colour translation is a method to augment the colour contrast of the input grey image, which can improve the accuracy of the brain segmentation performance (Li et al., 2017). The translation process is to obtain practical features and intensify the visual by mapping a single channel of the grey level pixel into Red, Green, and Blue channels (Afruz et al., 2010). 
Image segmentation is a method of separating an image into smaller regions with identical attributes. The attributes can be texture, greyscale value, brightness, shape, colour or texture (Tyan et al., 2014; Kipli \& Kouzani, 2015). The practice of image segmentation in medical is to provide meaningful information to the medical experts. K-means clustering is one of the popular methods under the clustering algorithm. This method divides or groups similar attributes of data points into one cluster and different attribute into different clusters.

In the subsequent subsections, we will present the proposed pseudo-colour conversion along with the clustering algorithm to segment acute ischemic stroke lesion in detail.

\section{The Proposed Segmentation Algorithm}

Initially, brain DWI images are gathered. Then, pre-processing operations are performed to intensify the quality of the images. Removal of non-brain tissues such as skull and bias field correction is proposed for this image. Subsequently, the colour space translation of an RGB colour map is applied, which contains Red, Green, and Blue values for each grey value item. The transformation converts the RGB image to the Commission on Illumination (CIE) Lab (L*a*b) colour model to rectify important features before undergoing the clustering process. The colour plane $\mathrm{L}^{*} \mathrm{a} * \mathrm{~b}$ calculates the tri-stimulus coefficient in terms of luminosity layer $\mathrm{L}^{*}$, a chromaticity-layer $\mathrm{a}^{*}$, and chromaticity-layer $\mathrm{b}^{*}$. This colour space specifies where the colour positioned along the red-green axis and blue-yellow axis. Both $\mathrm{a}^{*}$ and $\mathrm{b}^{*}$ channels include all essential colour information; hence, the colour information can be used as a classifier for clustering accommodate a region of interest. The histogram-based is utilized by the brightness attribute of $\mathrm{L}^{*}$ to exclude the pixels that are not related to the selected cluster. The difference between two layers $a^{*}$ and $b^{*}$ are calculated using the Euclidean distance metric. K-means clustering algorithm will find partitions, so that pixel values within each cluster are adjacent to each other based on the Euclidean distance metric obtained.

\section{Image Acquisition}

Images from one stroke patient (male, 60 years old) had been obtained in this research. DWI images were obtained on a 1.5T Siemens scanner from Sarawak General Hospital. With the following characteristics: repetition time $(\mathrm{TR})=4.40 \mathrm{~s}$; echo time $(\mathrm{TE})=80$ $\mathrm{ms}$; inversion time $=$ one acquisition; flip angle $=90^{\circ}$; field of view $(\mathrm{FOV})=229 \mathrm{~mm}$; 25 slices; and in-plane matrix $=130 \times 130$; slice thickness, $5 \mathrm{~mm}$; and gap between each slice, $6 \mathrm{~mm}$. Image resolution $=260 \times 260$. The sample had medical records that had been confirmed by a clinical neuroradiologist. For this study, three slices were giving meaningful information required.

In addition to that, Anatomical Tracings of Lesions After Stroke Release 1.1 or known as ATLAS R1.1 is an open-source dataset comprising of 304 images of T1-weighted MRIs 
neuroimaging was being utilized in this research for experiment testing. This dataset includes manually segmented stroke lesions as a gold standard. Image data were gathered on a 1.5 Tesla $(\mathrm{T})$ scanner with a resolution of $0.9 \mathrm{~mm} \times 0.9 \mathrm{~mm} \times 3.0 \mathrm{~mm}$.

\section{Image Pre-processing}

Before segmentation, image pre-processing is a process of removal of non-brain tissues and improves the features of images acquired from the scanners. Several pre-processing operations implemented for this approach comprise of skull stripping, and bias field correction. The raw DWI image had an 8-bit unsigned integer. Skull stripping or non-brain tissue removal is the procedure to eradicate the skull tissues from the brain images and acquired brain tissue before progressing to the segmentation. This procedure is a crucial process due to the forefront to obtain effective segmentation outcomes for precise diagnosis (Kalavathi \& Prasath, 2016). A mathematical morphology procedure is proposed to remove the non-brain tissue. Fundamentally, morphology operations that apply to this procedure are dilation and erosion. The image was converted to greyscale before progressing into the binary image while the structuring element used to erode the binary image was diskshaped with a radius of 55 .

Intensity inhomogeneity (IIH), also known as the bias field signal is a smooth and lowfrequency signal that distorts the quality of MR images during the acquisition process. The distorted signal produces a low-quality image, thus reduced the high-frequency information of the image, such as the intensity gradient of image pixels. The proposed method to correct the IIH was by employing the energy minimization optimization method. This method has proven to remove the bias field signal from the MRI images accurately (Li et al., 2014).

\section{Pseudo-colour Translation}

An 8-bit grayscale image of DWI would be translated into a pseudo-colour image with RGB value. The pseudo-coloured image was a single channel grayscale image that had colour assigned to it. In other words, the gray values of the image would match with the red, green, and blue values so that the shade of gray were displayed as colourized pixels. The translated image showed a pixel with a specified value of each colour. Therefore, the differences in colour value in the translated image reflected the changes in the intensity of the image. A formula to convert the grayscale image to RGB is shown below.

$$
\text { gray }=0.299 \mathrm{Red}+0.587 \text { green }+0.114 \text { blue }(\text { Khalil \& Ali, 2013) }
$$

Hence, the translated colour image then be transformed into a CIELab colour model to recover significant attributes. The luminance layer or intensity value of $\mathrm{L}$ of a greyscale image is represented with range 0 - 255 while a colour image utilized RGB coordinate 
system with Red-Green-Blue corresponded components. Thus, colour plane L*a*b will be computed into tristimulus components with the following Equation 1:

$$
\begin{aligned}
& \mathrm{W}=0.4303 R+0.3416 \mathrm{G}+0.1784 \mathrm{~B}, \\
& \mathrm{Y}=0.2219 \mathrm{R}+0.7068 \mathrm{G}+0.0713 \mathrm{~B}, \\
& \mathrm{Z}=0.0202 \mathrm{R}+0.1296 \mathrm{G}+0.9393 \mathrm{~B}
\end{aligned}
$$

The CIELAB colour model is computed as Equation 2

$$
\begin{aligned}
& L^{*}= 116\left(\mathrm{~h}\left(\mathrm{Y} / \mathrm{Y}_{\mathrm{s}}\right)\right)-16 \\
& a^{*}= 500\left(\mathrm{~h}\left(\mathrm{~W} / \mathrm{W}_{\mathrm{s}}\right)-\mathrm{h}\left(\mathrm{Y} / \mathrm{Y}_{\mathrm{s}}\right)\right) \\
& b^{*}=200\left(\mathrm{~h}\left(\mathrm{Y} / \mathrm{Y}_{\mathrm{s}}\right)-\mathrm{h}\left(\mathrm{Z} / \mathrm{Z}_{\mathrm{s}}\right)\right), \\
& \mathrm{h}(\mathrm{q})=\{\sqrt[3]{q}, \quad \mathrm{q}>0.008856 \\
&\{7.787 \mathrm{q}+16 / 116, \quad \mathrm{q} \leq 0.008856
\end{aligned}
$$

Where Ys, Ws, and Zs are the reference of the white point. Both $\mathrm{a}^{*}$ and $\mathrm{b}^{*}$ layers comprise all required colour information in red-green axis and blue-yellow axis, respectively. Hence, the Euclidean distance will be determined in the clustering process.

\section{K-means Clustering Algorithm}

In the proposed method, we applied the clustering algorithm to segment acute ischemic stroke lesion core. The process started with a few pre-processing steps to eliminate nonbrain tissues and bias field correction to enhance the image. The grey-level image of raw brain MRI was inadequate to display fine features. Thus, the pseudo-colour transformation was applied to acquire more useful information and intensify the visual.

$\mathrm{K}$-means clustering is a commonly utilized method of the clustering algorithm to separate data into a specific number of groups. The process was started by assigning data points to a group by computing the Euclidean distance among the data points, and the cluster centroid was at the lowest value. The homogeneous (similar) data points were achieved by obtaining less variation within the clusters. Euclidean distance can be calculated as in Equation 3,

$$
\mathrm{d}=\left\|\mathrm{x}_{\mathrm{i}}-\mathrm{c}_{\mathrm{j}}\right\|
$$

where $X=\{x i \mid i=1,2 \ldots, 1\}$ and $C=\{c j \mid j=1,2 \ldots, k\}$ while 1 is the clustered data and $\mathrm{k}$ is the cluster centroids. The resulting step is to recalculate the mean of the cluster centroids 
based on their cluster members and regroup the attribute corresponding to the new centroids. The criterion discontinued when all cluster centroids started to merge.

\section{Experimental Setup}

There are two experiments conducted to evaluate and validate the performance of the proposed segmentation algorithm. Experiment I was done by using diffusion weighted images (DWI) from the SGH datasets. These images were suggested by the clinical radiologist based on the location of the disease. The segmentation outcome was compared to the ground truth provided by a clinical radiologist. Experiment II is done by selecting 80 T1-weighted brains MRI from the Anatomical Tracings of Lesions After Stroke Release 1.1 dataset (Liew et al., 2018). The criteria for selecting these images were based on the location of acute stroke (left or right hemisphere of the brain) and the size of the acute stroke. The segmentation outcome was compared to the ground truth provided in the dataset.

The segmentation flow process is following the proposed process flow that has been discussed previously. A series of the segmentation process was from the image acquisition, pre-processing, segmentation, and performance evaluation would be carried out. Analysis and interpretation of the results will be conducted.

\section{Evaluation Metrics}

In this paper, the accuracy, specificity, sensitivity, and dice coefficient (DC) metrics to calculate the segmentation performance. The accuracy, specificity, sensitivity, and DC metrics are defined as Equation 4, 5, 6, and 7.

$$
\begin{aligned}
& \text { Accuracy }=\frac{\mathrm{TP}+\mathrm{TN}}{\mathrm{P}+\mathrm{N}}=\frac{\mathrm{TP}+\mathrm{TN}}{\mathrm{TP}+\mathrm{FP}+\mathrm{TN}+\mathrm{FN}} \times 100 \\
& \mathrm{DC}=\frac{2 \mathrm{TP}}{2 \mathrm{TP}+\mathrm{FP}+\mathrm{FN}} \\
& \text { Sensitivity }=\frac{\mathrm{TP}}{\mathrm{TP}+\mathrm{FN}} \\
& \text { Specificity }=\frac{\mathrm{TN}}{\mathrm{TN}+\mathrm{FP}}
\end{aligned}
$$

Where TP is a true positive, TN is a true negative, FP is a false positive, and FN is a false negative. The TP and TN are the numbers of correctly classified positive and negative classes by the segmentation algorithm. Whereas FP and FN are those images that are incorrectly classified. 
The segmentation of the acute ischemic lesions is essential in clinical diagnosis. Therefore, it is necessary to use lesion-wise metrics to assess segmentation performance. Accuracy is a system to measure the degree of closeness of measurements of a quantity compared to the ground truth. On the other hand, DC is a statistical validation system widely used in the MR image segmentation to measure the spatial overlap of the target regions (Zou et al., 2004). In this case, the automated segmentation image was compared with the ground truth provided by the clinical radiologist. Sensitivity refers to the outcome of the test to correctly detect the presence of the target lesions, while specificity relates to the rightly reject the existence of lesions.

\section{RESULTS AND DISCUSSION}

\section{Pre-processing}

In the proposed method, mathematical morphological operation and energy minimization optimization processes were applied to remove non-brain tissue and bias field correction, respectively. The outer layer of the skull, as observed in Figure 2, was successfully removed from the image (Figure 3). The removal of skull tissues is significant to improve the robustness of the segmentation process and influence the segmentation outcome. The bias field of DWI image had been effectively excluded from the raw image as exhibited in Figure 4. The different intensity value existed in an image might alter the segmentation process afterwards.

\section{Pseudo-colour Translation}

Figure 5 represents the image after a pseudo-colour translation. The greyscale image of an MRI was inadequate to support fine features; thus, pseudo-colour translation was applied to the image. The transformation mapped the 8-bit image with grey level values ranges $0-255$ into Red, Green, and Blue colour.

\section{Segmentation}

After the establishment of the pixel values range, the converted image was separated into RGB channels. Then, the RGB channels were translated into CIELab space to calculate the mean of $a^{*}$ and $b^{*}$ value. The clustering algorithm occurred to cluster the homogenous pixel values based on the Euclidean distance obtained. Figure 6 shows the segmented result after applying K-means clustering. The image obtained was following cluster selection in the K-means clustering algorithm, and region of interest was extracted successfully. Figure 7 displays the overlaid of the ROI against the original brain image. 


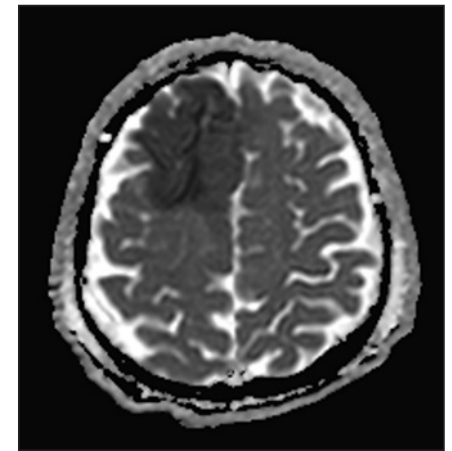

Figure 2. Raw image of DWI

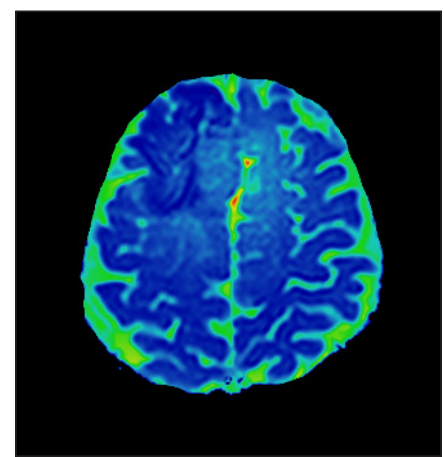

Figure 5. Image after pseudocolour translation

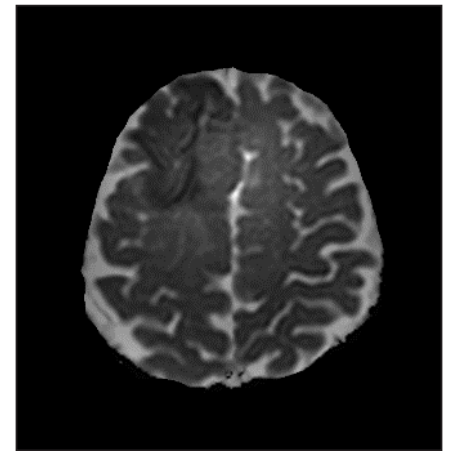

Figure 3. Skull stripped brain image

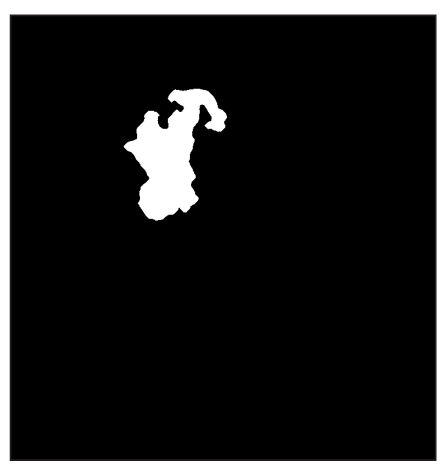

Figure 6. Segmented region of interest

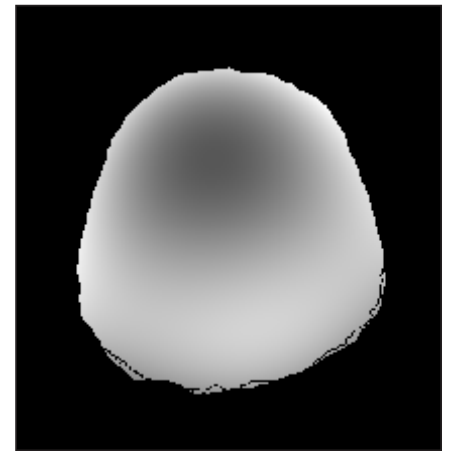

Figure 4. Bias field of an image

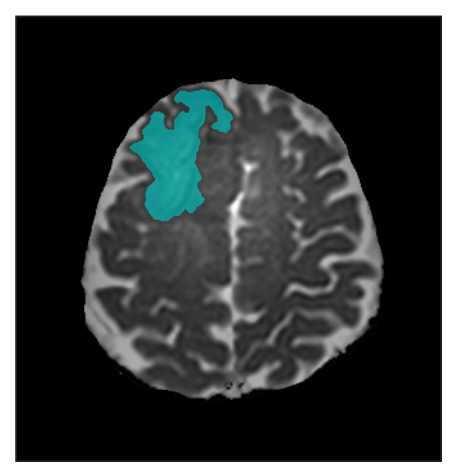

Figure 7. Overlaid of segmented ROI and brain image

\section{Performance Evaluation}

The segmentation algorithm was employed for Experiment I which were on DWI images. The performance evaluations metrics for this segmentation algorithm included accuracy, DC, specificity, and sensitivity.

Table 1 shows the achievement of the clustering algorithm for the three DWI images for different time-space. The results show accuracy, DC, specificity, and sensitivity. The maximum accuracy achieved was $90.080 \%$ indicating the proposed method was able to segment the acute ischemic stroke, while the average accuracy for three DWI images was $88.790 \%$.

From Table 1, Image 1 provides the lowest accuracy amongst the other two images by $86.820 \%$. The most insufficient accuracy achieved is due to the existence of high-intensity pixel incorporate into the region of interest and caused the over-segmentation and delivers high values in false positive with 0.194 compared with the remaining images.

In Experiment II, the dataset used was ATLAS dataset R1.1. The purpose of this experiment was to obtain the performances of the proposed segmentation algorithm tested on the several different images in the same dataset with the different sizes of the lesion, 
Table 1

Performance evaluation segmentation of the region of interest

\begin{tabular}{ccccc}
\hline Index & Accuracy (\%) & Dice Coefficient (DC) & Specificity & Sensitivity \\
\hline Image 1 & 86.820 & 0.848 & 0.194 & $\mathbf{0 . 0 3 0}$ \\
Image 2 & $\mathbf{9 0 . 0 8 0}$ & $\mathbf{0 . 8 9 0}$ & $\mathbf{0 . 0 7 1}$ & 0.133 \\
Image 3 & 89.460 & 0.882 & 0.140 & 0.058 \\
\hline Average & 88.790 & 0.873 & 0.135 & 0.074 \\
\hline
\end{tabular}

location of the lesion on the brain, and varying intensity of the lesion. MRI modality that was used in this simulation was $\mathrm{T} 1$-weighted $(\mathrm{T} 1 * \mathrm{w})$, whereas the location of the lesion was varied (left of right hemispheres) in each of the 80 selected brain images. The selection of these brain images was considered the location of the lesion on the brain image.

Accuracy, DC, sensitivity, and specificity evaluations were applied to compare the performance of the algorithm. Figure 8 and 9 give the boxplots of the segmentation algorithm performance in terms of accuracy, dice coefficient, sensitivity, and specificity. The boxplot represents the distribution of the performance of the 80 images from the ATLAS dataset. The boxplot exhibits the full range of variations (from min to max), and the middle line is the median. Boxplot is a systematic way of displaying the distribution of data based on the maximum, third quartile, median, first quartile, and minimum.

From Figure 8, the maximum accuracy achieved was $89.600 \%$. In comparison, the average accuracy was $74.090 \% \pm 1.805 \%$ with a $95 \%$ confidence level. The lowest accuracy attained was $53.17 \%$ due to the undistinguished intensity of the compressed image. The conversion of the pseudo-colour translation may affect the intensity of the image.

From Figure 9, the DC, sensitivity, and specificity of the segmentation algorithm were presented - DC of the algorithm ranging from 0.120 to 0.880 with a median of 0.630 . The $95 \%$ confidence interval calculated was \pm 0.038 . The lowest DC attained was 0.12 due to the distribution of contrast was inconsistent on the image. This condition caused the spatial overlap between the ground truth, and the segmented image was disturbed.

The maximum value sensitivity and specificity of this segmentation algorithm achieved were 1 , respectively and indicated that the actual positive values and negative values labelled from these images were accurately categorized. The $95 \%$ confidence interval revealed that the sensitivity and specificity mean values were $0.690 \pm 0.042$, and $0.780 \pm$ 0.023 , respectively. The sensitivity evaluation is to measure the ratio of positive voxels in the ground truth that was identified as positive by the segmentation. In contrast, evaluation of the specificity measures the ratio of negative voxels in the ground truth that is also identified as unfavorable by the segmentation (Taha \& Hanbury, 2015).

The proposed methodology requires uncomplicated pre-processing and colour space translation procedures before undergoing a segmentation task. The segmentation algorithm in this work is easily understood. The overall implementation procedure for the 
Abang Mohd Arif Anaqi Abang Isa, Kuryati Kipli, Ahmad Tirmizi Jobli, Muhammad Hamdi Mahmood, Siti Kudnie Sahari, Aditya Tri Hernowo and Sinin Hamdan

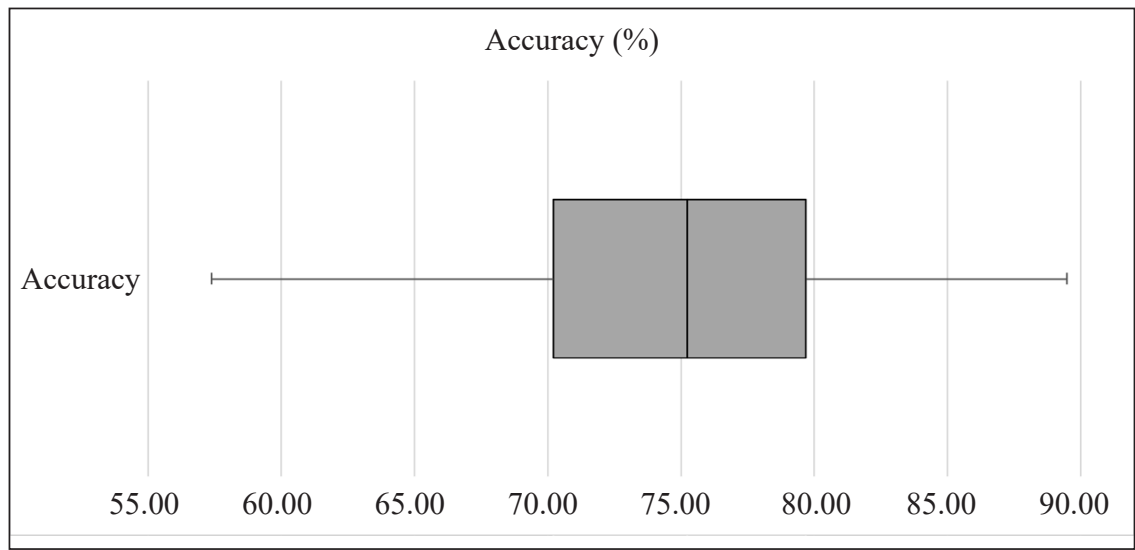

Figure 8. Boxplot of the average segmentation accuracy results

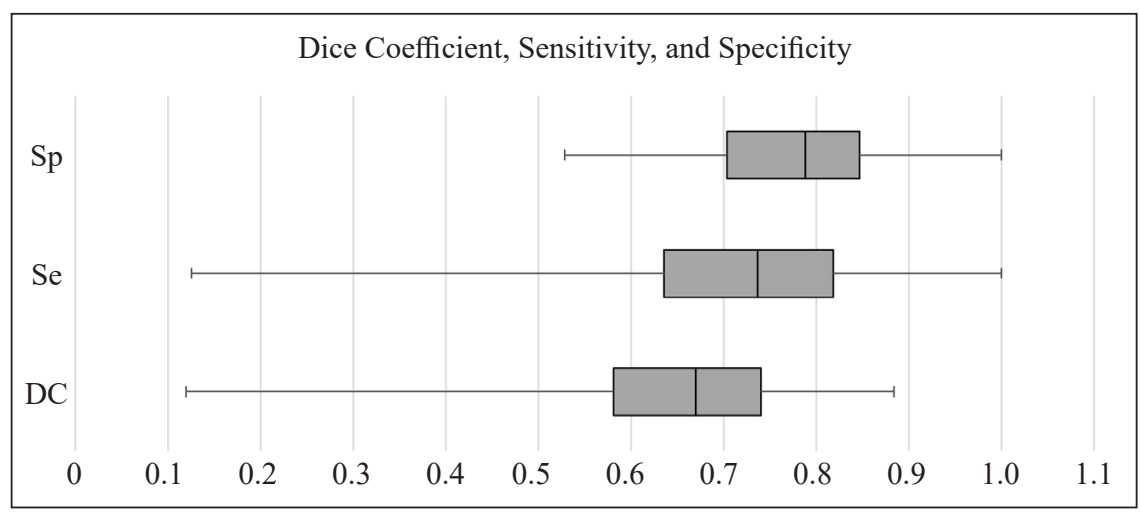

Figure 9. Boxplot of dice coefficient, sensitivity and specificity of the segmentation results

segmentation of the acute stroke lesion region is simple and straight forward. Our work yield the maximum accuracy of $90.080 \%$ on diffusion weighted image and $89.600 \%$ on T1-weighted image respectively. In addition, maximum dice coefficient achieved from our work were 0.890 and 0.880 for DWI and T1-weighted, respectively. Experimentally, our method exhibited that the proposed segmentation techniques deliver good accomplishment, and the results at par with the existing works (Muda et al. 2017, Nag et al. 2017, Vupputuri et al 2017, and Gautam \& Raman, 2019).

The implementation of pseudo-colour segmentation on the grayscale image significantly affect the quality of an image understanding system (Chen \& Wang, 2004). The images were segmented into homogeneous areas with similar spectrum information. In our findings, the colour homogeneity was based on the mean value calculated from $\mathrm{a}^{*}$ and $\mathrm{b}$ * channels from CIELAB colour model. This shows that the spectral information of the image is crucial in imagery data, and the performance of the clustering segmentation significantly increases. While the $\mathrm{L}^{*}$ layer separate the dark and light coloured object. The $\mathrm{L}^{*}$ layer comprises 
the brightness values of each colour and the brightness value of the image was extracted in the clustering algorithm. The extraction of the brightness value reduces the number of iterations in the clustering algorithm. Hence, colour based image segmentation integrate in the clustering reduce the computational cost avoiding pixel features calculation in the image.

This paper proposed a segmentation algorithm based on structural brain MRI that plausibly aid the assessment of the acute ischemic stroke lesion. This proposed system is a ground-breaking approach that able to delineate stroke lesion precisely and delivers the second opinion to the specialist. The automated segmentation of the lesion is a challenging task because of the presence of artefacts, bias field, and brain skull during the acquisition process. In contrast, manual segmentation is a subjective, labour-intensive and varied in results depending on the specialists' expertise, whereas the automated segmentation.

An automated segmentation algorithm for brain MRI is developed by employing pseudo-colour conversion and clustering algorithm. The proposed segmentation algorithm involves a series of pre-processing, pseudo-colour translation and clustering processes. Each step in this image segmentation carries significant contributions towards the outcome. For instance, pre-processing steps was to reduce the miscalculation and error during the estimation of the region of interest. The occurrence of non-uniform grey-level intensities can reduce the segmentation accuracy. At the same time, the pseudo-colour translation is to enhance the visualization of the different brain region, namely, lesion, white matter, grey matter, and cerebrospinal fluid. Finally, the details of the pixel in RGB colour channels are clustered according to the resemblance by employing the K-means clustering algorithm.

\section{CONCLUSION}

In this research, we employed the pseudo-colour method on the greyscale DW image to intensify the visual perception. The proposed segmentation algorithm had shown a promising result when applied to the brain DW image focusing on the hyperintense lesions. Pre-processing steps were conducted for skull stripping and bias field correction. The proposed segmentation algorithm was assessed and compared against the ground truth provided by the clinical neuroradiologist. The result showed that the maximum accuracy achieved was $90.08 \%$ and capable of segmenting the acute ischemic stroke from brain DWI images successfully. This proposed segmentation algorithm hopefully will help medical experts to differentiate the lesion with the healthy brain tissues. Based on the obtained results, this research potential should be validated using the larger dataset to draw a more concrete conclusion. To achieve these improvement, cooperative advancement across several institutions to form larger dataset of acute ischemic stroke based on brain MR images for efficient development of detection algorithm. Besides, depending on a single modality of MRI is not sufficient to establish a complete acute ischemic stroke thrombolysis. Hence, combining the information from multiple MRI sequences may be helpful. 


\section{ACKNOWLEDGEMENTS}

We want to thank the Sarawak General Hospital (SGH) for making the brain MRI data available. The first author would like to acknowledge Yayasan Sarawak and Universiti Malaysia Sarawak (UNIMAS) for financial support through the Yayasan Tun Taib scholarship.

\section{REFERENCES}

Afruz, J., Wilson, V., \& Umbaugh, S. E. (2010). Frequency domain pseudo-colour to enhance ultrasound images. Computer and Information Science, 3(4), 24-34.

Chen, H. C., \& Wang, S. J. (2004). The use of visible color difference in the quantitative evaluation of color image segmentation. In 2004 IEEE International Conference on Acoustics, Speech and Signal Processing (Vol. 3, pp. 3-593). IEEE Conference Publication. https://doi.org/10.1109/ICASSP.2004.1326614

Dhankar, S., Tyagi, S., \& Prasad, T. V. (2010). Brain MRI segmentation using K-means algorithm. In National Conference on Advances in Knowledge Management, NCAKM 2010 (pp. 1-5). Lingaya's University. https://doi.org/10.13140/RG.2.1.4979.0567

Gautam, A., \& Raman, B. (2019). Segmentation of ischemic stroke lesion from 3D MR images using random forest. Multimedia Tools and Applications, 78(6), 6559 - 6579. https://doi.org/10.1007/s11042-018-6418-2

Gonzalez, R., \& Schwamm, L. (2016). Imaging acute stroke. In Handbook of clinical neurology, (pp 293-315). Elsevier's ScienceDirect. https://doi.org/10.1016/B978-0-444-53485-9.00016-7

Graves, M. J., \& Mitchell, D. G. (2013). Body MRI artifacts in clinical practice: A physicist's and radiologits' perspective. Journal Magnetic Resonance Imaging, 38(2), 269-287. https://doi.org/10.1002/jmri.24288

Jinlong, H. U., Xianrong, P., \& Zhiyong, X. U. (2012). Study of grey image pseudo-colour processing algorithms. In International Symposium on Advanced Optical Manufacturing and Testing Technologies: Large Mirrors and Telescopes. (Vol. 8415, p. 841519). International Society for Optics and Photonics. https://doi.org/10.1117/12.977197

Kalavathi, P., \& Prasath, V. B. S. (2016). Methods on skull stripping of MRI head scan images - A review. Journal of Digital Imaging, 29(3), 365-379. https://doi.org/10.1007/s10278-015-9847-8

Khalil, Y. A., \& Ali, P. J. M. (2013). A proposed method for colorizing grayscale images. International Journal of Computer Science and Engineering, 2(2), 109-114.

Kipli, K., \& Kouzani, A. Z. (2015). Degree of contribution (DoC) feature selection for structural brain MRI volumetric features in depression detection. International Journal of Computer Assisted Radiology and Surgery, 10(7), 1003-1016. https://doi.org/10.1007/s11548-014-1130-9

Krupa, K., \& Bekiesińska-Figatowska, M. (2015). Artifacts in magnetic resonance imaging. Polish Journal of Radiology, 80, 93-106. https://doi.org/10.12659/PJR.892628

Li, C., Gore, J. C., \& Davatzikos, C. (2014). Multiplicative intrinsic component optimization (MICO) for MRI bias field estimation and tissue segmentation. Magnetic Resonance Imaging, 32(7), 913-923. https://doi. org/10.1016/j.mri.2014.03.010 
Li, H., Chen, C., Feng, S., \& Zhao, S. (2017). Brain MR image segmentation using NAMS in pseudo-colour. Computer Assisted Surgery, 22(0), 170-175. https://doi.org/10.1080/24699322.2017.1389395

Liew, S. L., Anglin, J. M., Banks, N. W., Sondag, M., Ito, K. L., Kim, H., \& Stroud, A. (2018). A large, opensource dataset of stroke anatomical brain images and manual lesion segmentations. Scientific data, 5(1), 1-11. https://doi.org/10.1038/sdata.2018.11

Merino, J. G., \& Warach, S. (2010). Imaging of acute stroke. Nature Reviews Neurology, 6(10), 560-571. https://doi.org/10.1038/nrneurol.2010.129

Muda, A. F., Saad, N. M., Abu Bakar, S. A. R., Muda, S., \& Abdullah, A. R. (2017, March 15-17). Automated stroke lesion detection and diagnosis system. In Proceedings of the International MultiConference of Engineers and Computer Scientists. Hong Kong.

Nag, M. K., Koley, S., China, D., Sadhu, A. K., Balaji, R., Ghosh, S., \& Chakraborty, C. (2017). Computerassisted delineation of cerebral infarct from diffusion-weighted MRI using Gaussian mixture model. International Journal of Computer Assisted Radiology and Surgery, 12(4), 539-552. https://doi. org/10.1007/s11548-017-1520-x

Nitta, G. R., Sravani, T., Nitta, S., \& Muthu, B. (2020). Dominant gray level based K-means algorithm for MRI images. Health and Technology, 10, 281-287. https://doi.org/10.1007/s12553-018-00293-1

Purushotham, A., Campbell B. C. V., Straka, M., Mlynash, M., Olivo, J., Bammer, R., Kemp, S. M., Albers, G. W., \& Lansberg M. G. (2015). Apparent diffusion coefficient threshold for delineation of ischemic core. International Journal of Stroke, 10(3), 348-353. https://doi.org/10.1111/ijs.12068

Taha, A. A., \& Hanbury, A. (2015). Metrics for evaluating 3d medical image segmentation: Analysis, selection, and tool. BMC Medical Imaging, 15(29), 1-28. https://doi.org/10.1186/s12880-015-0068-X

Tyan, Y. S., Wu, M. C., Chin, C. L., Kuo, Y. L., Lee, M. S., \& Chang, H. Y. (2014). Ischemic stroke detection system with a computer-aided diagnostic ability using an unsupervised feature perception enhancement method. International Journal of Biomedical Imaging, 2014, 1- 24. https://doi.org/10.1155/2014/947539

Vupputuri, A., Ashwal, S., Tsao, B., Haddad, E., \& Ghosh, N. (2017). MRI based objective ischemic corepenumbra quantification in adult clinical stroke. In Proceedings of the Annual International Conference of the IEEE Engineering in Medicine and Biology Society (pp. 3012-3015). IEEE Conference Publication. https://doi.org/10.1109/EMBC.2017.8037491

Zou, K. H., Warfield, S. K., Bharatha, A., Tempany, C. M., Kaus, M. R., Haker, S. J., Wells, W. M., Jolesz, F. A., \& Kikinis, R. (2004). Statistical validation of image segmentation quality based on a spatial overlap index. Academic Radiology, 11(2), 178-189. https://doi.org/10.1016/S1076-6332(03)00671-8 
